\title{
Overactive bladder in the vulnerable elderly
}

\author{
This article was published in the following Dove Press journal: \\ Research and Reports in Urology \\ 3 October 2014 \\ Number of times this article has been viewed
}

\section{Gillian F Wolff' \\ George A Kuchel ${ }^{2}$ \\ Phillip P Smith ${ }^{1,2}$ \\ 'Division of Urology, Department of Surgery, ${ }^{2}$ UConn Center on Aging, University of Connecticut Health Center, Farmington, CT, USA}

\begin{abstract}
Overactive bladder $(\mathrm{OAB})$ is a common problem that may occur in individuals of all ages. It has a considerable impact on patient quality of life, and although moderately effective management strategies do exist, this condition often remains undiagnosed and untreated. OAB needs to be viewed as a symptom complex. Its presentation and management are complicated in the vulnerable elderly by the presence of baseline frailty and multiple coexisting chronic conditions. Furthermore, and beyond a simple understanding of symptomatology, providers must address patient goals and motivations as well as the expectations of caretakers. These multiple levels of perception, function, expectations, and treatment efficacy/risks must be tailored to the individual patient. While the vulnerable elderly patient may often have evidence of urinary tract dysfunction, $\mathrm{OAB}$ and urge urinary incontinence in this population must be understood as a multifactorial geriatric syndrome and viewed in the context of medical and functional baseline and precipitating risk factors. Expectations and goals must be tailored to the resources of vulnerable elderly patients and their caregivers, and care must be coordinated with other medical care providers. The management of OAB in the vulnerable elderly often poses significant management challenges. Nonetheless, with a thoughtful approach and an aim towards future research specifically for this population, significant reductions in morbidity and mortality long with enhancement in health-related quality of life are possible.
\end{abstract}

Keywords: urinary incontinence, urgency, antispasmodics, aging, frailty

\section{Introduction}

Urinary symptoms such as urgency/frequency and incontinence become increasingly prevalent with aging. ${ }^{1,2}$ A large multinational population-based survey estimated the prevalence of overactive bladder (OAB) in Europe and Canada to be $12.8 \%$ in women and $10.8 \%$ in men. ${ }^{2}$ The NOBLE (National Overactive Bladder Evaluation) study showed similar trends in the USA, with OAB being twice as prevalent in individuals over 65 years of age than in those aged 45 years or younger. ${ }^{3}$ Urinary symptoms have a considerable negative impact on quality of life and health in the elderly, ${ }^{4,5}$ and have been shown to be associated with increased risk of falls and fractures. ${ }^{6,7}$ Costs associated with urinary symptoms in the aged are significant. ${ }^{8,9}$ With the aging of the population, it is estimated that by 2025 there will be 52 million adults in the USA with lower urinary tract symptoms. ${ }^{10}$ Thus, the burden of these symptoms on society is increasing.

Even in the frail elderly, the morbidity associated with OAB can be ameliorated to a large degree with appropriate evaluation and treatment. The frail elderly are by definition a particularly vulnerable population, so evaluation and treatment of lower
Correspondence: George Kuchel
UConn Center on Aging, University of Connecticut Health Center,

263 Farmington Avenue, MC-5215,

Farmington, CT 06030-5215, USA

Tel +l 8606793956

Fax + I 860679 I307

Email kuchel@uchc.edu 
urinary tract symptoms must be approached with special caution. Even in the general population, pathways for evaluation and available treatments can be controversial and are often of less than ideal effectiveness. Given that urinary disorders in the aged are often compounded by multiple medical comorbidities, clinical paradigms appropriate for a more general population can be further compromised. While there is a dearth of level 1 evidence for interventions in the frail elderly, and clinical trials rarely include this population, we present a review of evaluation and treatment of OAB in the vulnerable elderly patient in the context of a geriatric syndrome.

\section{Overactive bladder}

$\mathrm{OAB}$ is characterized by urinary frequency/urgency and is often associated with urinary incontinence, and is defined by the International Continence Society as "urgency, with or without urge incontinence, usually with increased frequency and nocturia". ${ }^{11}$ Thus, OAB is a symptom complex, and not a diagnosis with specific pathophysiologic implications. In younger populations, a detailed evaluation of OAB symptoms is frequently unproductive, so the OAB complex assumes the significance of a diagnosis, with resultant treatment with therapies nominally aimed at "OAB". Recommended pathways for diagnosis and treatment of $\mathrm{OAB}$ in the general population have recently been published by the American Urological Association. ${ }^{12}$ However, in specific populations, such as the vulnerable elderly, these guidelines may not be as applicable. In these situations, it may be more appropriate to view $\mathrm{OAB}$ as its defined symptom complex, and base treatment on a more global diagnostic evaluation, extending beyond a bladder-centric focus.

The American Urological Association/Society of Urodynamics, Female Pelvic Medicine and Urogenital Reconstruction guidelines for the diagnosis and treatment of OAB recommend as part of the initial diagnostic workup a history, physical examination, and urinalysis to specifically characterize a patient's OAB symptoms and exclude other disorders that could potentially cause similar symptoms. The history should include information on voiding frequency, fluid intake, comorbid conditions, and medications. The physical examination should include an evaluation of cognitive function. Treatment should first include education and behavioral therapy, and most importantly set feasible goals for the specific patient. The importance of setting feasible goals and avoiding potentially unsafe or futile therapy is particularly relevant when caring for the frail elderly.

The etiology of OAB should be considered in all cases; however, the extent of evaluation and therapeutic intervention is highly specific to the individual patient and even moreso in the aged individual. OAB is a perceptual syndrome related to control of bladder volumes. ${ }^{13}$ Normal control of bladder volumes requires maintaining a low pressure, a capacious reservoir of urine in the bladder, the ability to relax the sphincteric mechanism and contract the detrusor smooth muscle to achieve voiding at an appropriate time and place, and the central abilities to perceive and control these relationships. Symptoms of OAB represent the perception of deficiencies in one or more of these areas. Unfortunately, symptoms often do not provide meaningful insight about the specific functional disorder or combination of disorders, hence the recommendations for some diagnostic evaluation prior to recommending treatment.

\section{Vulnerable or frail elderly}

Aging is associated with a continual (and not necessarily linear) decline in the person's ability to compensate for the vast range of stressors imposed by interaction of the environment with the impact of aging on systems physiology and biology at the level of individual organs or tissues. The ability to provide compensation, to maintain a steady internal milieu, and remain free of functional and symptomatic disorders is known as "homeostasis". In that context, the impact of aging with declines in the ability to robustly respond to such challenges has been termed "homeostenosis". At the point at which the impact of homeostenosis becomes the dominant feature of the individual's health, the impact of aging becomes highly evident. In particular, the vulnerable elderly are considered to be those older individuals in whom the sum of their homeostatic failures reaches the point at which they are at increased risk of notable functional decline or death in the near future. ${ }^{14}$ This definition would encompass the majority of individuals who could be considered as being frail or vulnerable as a result of a specific frailty phenotype and/or the accumulation of various deficits and comorbidities.

Consistent with its status as a symptom complex, there is no singular cause of OAB. In general, symptoms of OAB represent the perception of impaired urine storage. In younger patients, this perception may be frequently accurate; however, in the elderly individual, disorders of voiding and perceptual defects should also be considered. The impact of other morbidities, environmental factors, drugs the patient may be taking, prior surgeries, and other factors must all be considered in both the evaluation and treatment of OAB. ${ }^{15}$

Standard practice paradigms assume that the patient presents to the doctor with a list of symptoms, the doctor 
then tries to determine the underlying pathophysiology of those symptoms, makes a diagnosis, and finally recommends therapy based on that diagnosis. The multifactorial nature of frailty and other common geriatric syndromes renders this formulation inadequate, and an adherence to such a standard paradigm in the vulnerable elderly risks needless intervention, application of misdirected diagnostic and therapeutic interventions, unnecessary costs, and in the worst case iatrogenic morbidity or mortality.

\section{Evaluating OAB in the vulnerable elderly}

Rather than a focus on lower urinary tract pathology, the evaluation and care for urinary disorders in the elderly must have a broader focus, placing the functional disturbance in the context of the patient's overall situation. The patient experiences symptoms, there may be demonstrable physiologic disturbances, and underlying disorders relating to the symptoms may be morbid. These three concepts may overlap in individuals at risk for $\mathrm{OAB}$, but are not necessarily concurrent (see Figure 1). Especially in the vulnerable elderly, the impact and necessity of defining and addressing each of these domains must be carefully considered. Evaluation of OAB symptoms in the vulnerable elderly, moreso perhaps than in younger patients, requires that several questions be specifically addressed, as outlined in the following sections.

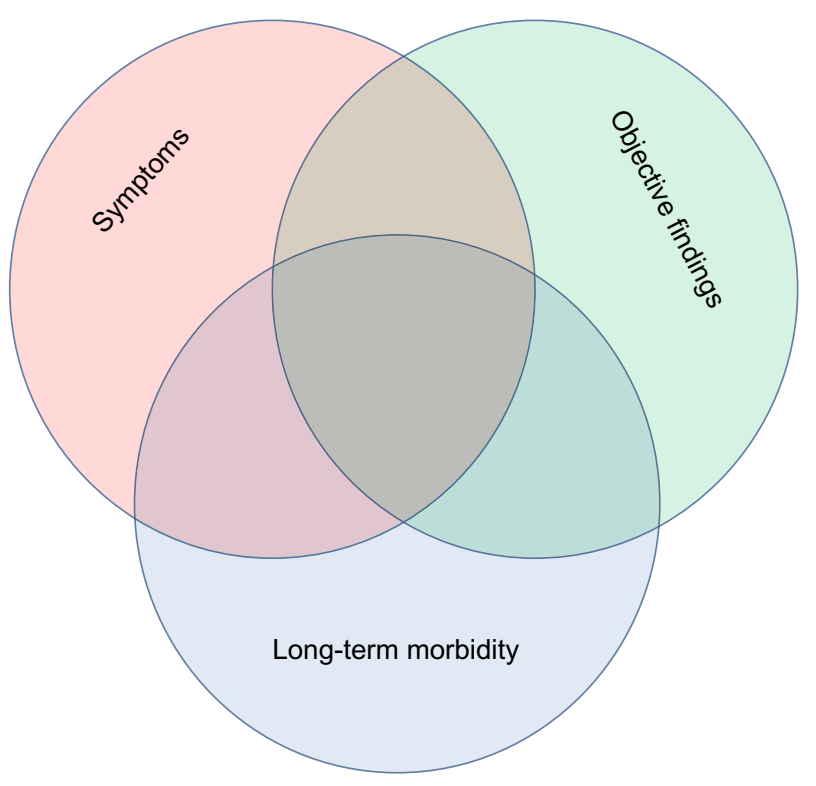

Figure I Conceptual relationship of clinical factors.

Notes: Symptoms, measurable function, and morbidity related pathophysiology, their evaluation and any treatments are related concerns which must be identified, clarified, and prioritized. While this sometimes-subtle distinction is always important, it assumes greater importance in the vulnerable elderly.

\section{Who is the patient?}

The assumption that the individual experiencing symptoms is necessarily the patient may not be true in the vulnerable elderly. These are often patients who have accommodated significant limitations in their functional capacities and abilities, but for whom care related to incontinence of urine, frequent trips to the bathroom, costs of supplies etc, become burdensome. In many, if not most, cases the patient will be the symptomatic individual, presenting with the classic OAB syndrome. Evaluation and treatment will follow, with the discussed limitations relative to published recommendations.

Furthermore, in some cases, the family, the nursing home, and the payor may all actually require the provider's attention, each with their own specific (and nonmedical) concerns related to the vulnerable elderly patient. It is incorrect to assume that the elderly patient with incontinence or urinary frequency necessarily suffers from "OAB", since the patient may actually not complain of any perception of disordered bladder control. In these cases, an assessment of practically available therapies may dictate the diagnostic evaluation. For example, a detailed urodynamic evaluation of the high-risk frail elderly patient with incontinence and a very high postvoid residual bladder volume might be hard to justify in the absence of reversible or treatable predisposing factors. This patient may be best served by clean intermittent catheterization, with the possible addition of pharmacologic intervention aimed at reducing incontinence due to detrusor overactivity, or an indwelling catheter. Both options have certain limitations and risks, but these decisions must be individualized and may be dictated by the nursing home, which may not have the staffing levels necessary to provide clean intermittent catheterization services.

\section{What are feasible treatment options?}

In younger patients with $\mathrm{OAB}$, the guidelines for treatment follow a stepwise and increasingly invasive pathway of evaluation and treatment. The basic evaluation of all patients includes a history and physical examination aimed at discovering obvious structural, infectious, or neurologic causes of OAB symptoms without generally resorting to expensive and potentially invasive technologies. When simple behavioral or pharmacologic treatment is inadequate, more invasive and costly evaluations are indicated to further pursue specific causes of the symptoms. However, in the vulnerable elderly, altered priorities, functional decline, and diminished ability to withstand invasive treatments may render some evaluation pathways unnecessary. 


\section{What are the consequences of inaction?}

Figure 1 illustrates the clinical observation that, in all patients, but especially the frail elderly, symptoms and demonstrable physiologic abnormalities may occur independently of each other. With or without symptoms, associated clinical conditions and disorders can contribute to longer-term morbidity stemming from OAB. For example, an elderly man with OAB symptoms and mild renal failure may have a chronically obstructed bladder resulting in high-pressure urine storage. These considerations increase his risk of worsening renal failure and ultimate death. The physician consulted for this patient's $\mathrm{OAB}$ must consider the importance of resolving the high-pressure urine storage problem and then, if desirable, consider the feasibility of bypassing the bladder via indwelling catheterization or trying to correct the bladder storage problem with pharmacologic or surgical means. There is little point in performing an extensive evaluation on this patient if it is known a priori that an assessment of competing risks indicates that the risk of renal failure is insignificant relative to the patient's other morbidities and/or life expectancy. Furthermore, even if avoidance of renal failure is desired, clinical assessment of costs and benefits may favor the institution of long-term passive drainage by indwelling catheter rather than the risks and costs of urodynamic evaluation and the subsequent potential for more invasive therapeutic considerations.

For the symptomatic patient, the question is more obvious and should be considered early on in the investigation. If symptom control is of greater importance than preventing long-term morbidity, the diagnostic and therapeutic focus can then be simplified. Failure to empty a capacious bladder is rare but can generally be managed with a catheterization regimen. A bladder incapable of volume storage without leakage is even less common, but can be managed by oral pharmacologic agents, neuromodulation, botulinum toxin (if appropriate and tolerated), or by indwelling catheter. If prevention of morbidity, which may emerge in the limited life expectancy of the medically vulnerable person is important, a more thorough diagnostic evaluation including urodynamics may be warranted, allowing a more directed application of available therapies.

\section{What is a reasonable diagnostic evaluation, given these concerns?}

The conclusion of the above questions is that available guidelines such as those promoted for a general symptomatic population must be tempered for the vulnerable elderly, taking into account patient expectations, homeostatic frailty, comorbidities, and expected life expectancy. Given the heterogeneity and frailty of this population, the approach to evaluation of OAB needs to be highly individualized. Nonetheless, in the context of the above concerns, a few overarching principles need to be considered during the evaluation, as follows.

\section{What other comorbidities may be contributing to the patient's symptoms?}

As a rule, $\mathrm{OAB}$ and urinary incontinence are not the sole genitourinary or other pathologies present in each vulnerable elderly patient. It is essential to address potentially treatable, reversible conditions. Wagg et al summarized common conditions contributing to urinary symptoms in the frail elderly by the mnemonic DIPPERSA (Delirium, Infection, Pharmaceuticals, Psychological, Excess urine output, Reduced mobility, Stool impaction, and Avoid treatment of asymptomatic bacteriuria). ${ }^{16}$ While this mnemonic was created specifically to address urinary incontinence, it is reasonable to apply it to OAB symptoms as well.

\section{What is the bladder's volume operating range?}

In this context, it is important to assess the patient's bladder capacity and post-void residual, and the difference between these volumes. Evaluating these volumes will help elucidate whether the primary problem is one of impaired urine storage or inadequate bladder emptying.

\section{What is the total urine production per day?}

The polyuric patient may report frequency and possibly incontinence even with a fully functional lower urinary tract. In a patient with a compromised lower urinary tract, polyuria will exacerbate any underlying pathology. In both cases, efforts at addressing the cause of polyuria may be the most effective therapy for amelioration of the symptoms. Alleviating the added stressor to the lower urinary tract could potentially make the lower urinary tract pathology manageable in itself, or at least allow further therapies to be more effective.

\section{What is the patient's cognitive status?}

The impact of impaired cognition on urinary function undoubtedly extends beyond the integrity and accuracy of urinary perceptions. Emerging evidence suggests that urinary control represents in many ways a failure of integrative brain processes. ${ }^{15}$ Adequacy in other functional domains such as mobility also impacts directly on urinary function, urinary perceptions, and the ability to maintain continence. 
Historic and objective anatomic and physiologic data from the cognitively impaired patient may not have the same implications as those from the cognitively intact patient. Brain imaging studies link degenerative changes (eg, white matter hyperintensities) in areas relevant to bladder sensory function and control to the patient's perception of more severe symptoms, but not necessarily a greater prevalence of symptoms or dysfunction. ${ }^{17,18}$ Similarly, the expected range of responses, including adverse events as well as the ability to sense, process, and respond to the sensation of urgency, may be different than in the cognitively intact individual. ${ }^{19}$ These uncertainties suggest that while the vulnerable elderly are potential candidates for the full range of evaluative and therapeutic interventions, considerable caution and individualized decision-making is necessary.

\section{What other health care professionals are involved with this patient?}

The vulnerable elderly almost by definition will interact with many different health care practitioners. A wide range of therapies, including medications, will be considered in the evaluation and management of OAB. Thus, it is important that the physician evaluating and treating $\mathrm{OAB}$ in the vulnerable elderly communicate with (and expect communication from) the patient's other health care providers.

\section{Treatment of OAB in the vulnerable elderly Behavioral modification and pelvic floor therapy}

In most patients, including the vulnerable elderly, first-line treatment for $\mathrm{OAB}$ consists of lifestyle and behavioral modifications. Becher et al identified four elements of nonpharmacologic therapy specifically targeted at urinary incontinence in older adults, ${ }^{20} \mathrm{ie}$, communication, behavior, training, and supportive measures. Specifically, the patient (and/or the appropriate caregiver) should be provided with education about the condition and the various treatment options. Behavioral interventions include bladder training, timed voiding, and habit training. The bladder symptoms themselves can be modified by bladder training and delayed voiding. Training refers specifically to pelvic floor muscle training and techniques for urge suppression. While most often applied in the context of stress urinary incontinence, pelvic floor muscle training has also been recommended for urge incontinence and $\mathrm{OAB}$ by both the European Association of Urology and the American Urological Association. ${ }^{12,21}$
Supportive measures include physiotherapy, such as biofeedback or percutaneous tibial nerve stimulation. However, it is important to note that patient cooperation is required, and participation by some vulnerable elderly patients may be limited in each of the above-mentioned nonpharmacologic approaches due to possible mobility, motivational, or cognitive impairments.

\section{Antimuscarinics}

Following evaluation and intervention for correctable causative pathology and a trial of behavioral therapy, drug therapy can be considered. First-line pharmacologic therapy generally consists of trials of antimuscarinic medications. The efficacy and tolerability of antimuscarinic drugs depends on their interactions with specific muscarinic receptor subtypes $\left(\mathrm{M}_{1}-\mathrm{M}_{5}\right)$, and the drug's ability to penetrate the blood-brain barrier. The effects of antimuscarinics on the lower urinary tract are thought to be mediated by the M2 and, mostly, M3 subtypes. These two subtypes are also thought to be responsible for unwanted antimuscarinic side effects, including dry mouth, constipation, and blurred vision. Especially concerning in the frail elderly is the potential cognitive impairment associated with antimuscarinics. This impairment is mediated via the $\mathrm{M} 1$ receptor in the central nervous system. The cognitive effects of these drugs may also be related to their ability to cross the blood-brain barrier and interact with the M1 receptor.

Many concerns have been raised regarding the use of antimuscarinic medications in the frail elderly. The frail elderly patient is by definition vulnerable, and small insults to cognition may be particularly pronounced and detrimental in this population. Compounding this is the fact that many of the frail elderly are already taking other medications for their comorbid conditions. Polypharmacy has the potential to interact with the metabolism of these drugs, and other medications may themselves have antimuscarinic properties, leading to increased exposure and increased risk of antimuscarinic side effects. ${ }^{22}$

Nevertheless, there is a dearth of level 1 evidence for the efficacy and tolerability of antimuscarinic therapy in the frail elderly. Older individuals, especially those with the multiple comorbidities that characterize the frail elderly, tend to be excluded from clinical trials. Most of the available data is extracted from post hoc subanalyses of trials with a limited number of patients over 65 years, and even fewer over 75 years. The few published prospective trials of antimuscarinics in older patients tend to exclude medically complex vulnerable elderly individuals, who generally have 
a higher disease burden and are more vulnerable to adverse events, yet conversely, may be more likely to gain the greatest benefit from therapy.

The paradigm underlying antimuscarinic therapy focuses on diminishing detrusor motor activity during filling. Therefore, relative to antimuscarinic therapy, the ideal molecule would be highly specific for M3 detrusor receptors. Given the particular sensitivity of the vulnerable elderly to misdirected pharmacologic effects, the more recently available agents have sought to minimize adverse events by more specific bladder targeting. For example, unlike the other available agents, trospium chloride is a quaternary amine, so should not cross the blood-brain barrier. Diminished penetration of the central nervous system has been demonstrated ${ }^{23}$ and hypothetically this should minimize the cognitive impact of this agent, but this has not been conclusively demonstrated in clinical experience. ${ }^{24,25}$ Darifenacin was proposed to specifically target bladder M3 receptors, thereby minimizing muscarinic side effects attributable to nonbladder M3 receptors as well as M1 and M2 receptors. ${ }^{26}$ Further, limited data suggest a favorable effect on cognitive function in older patients. ${ }^{27}$ However, evaluation of cognitive function has been tested only in healthy (not frail/vulnerable) older patients and therefore likely not generalizable to the vulnerable elderly. There is little comparative clinical evidence to suggest an advantage of any of the available agents other than oxybutynin. ${ }^{25,28}$

Recently, Dubeau et al published the first randomized controlled trial of antimuscarinic therapy for urge urinary incontinence in older community-dwelling patients with complex medical comorbidities and polypharmacy generalizable to the vulnerable elderly population. ${ }^{29}$ These authors performed a 12-week, randomized, double-blind, flexible-dose, placebo-controlled trial of fesoterodine in vulnerable elderly subjects with urge urinary incontinence. Subjects receiving flexible-dose fesoterodine had a significant reduction in their primary outcome measure, ie, number of urge urinary incontinence episodes per 24 hours from baseline to week 12 when compared with the placebo group. The fesoterodine group also had significant reductions in micturitions, daytime and night-time urgency episodes, and higher diary dry rates. The fesoterodine group also reported significantly higher satisfaction rates with their bladder condition and with therapy.

Fesoterodine had a safety profile similar to that seen in trials with younger healthier subjects. The most common adverse events were dry mouth and constipation. There was no decrease in mean Mini-Mental State Examination scores in patients randomized to either group. Three patients in the treatment arm did have a reduction in their self-reported mental status compared with none in the placebo arm. This occurred at the $8 \mathrm{mg}$ dose in two patients. However, about half of the patients chose to increase their dose from $4 \mathrm{mg}$ to $8 \mathrm{mg}$ and remained at that dose. This led the authors to recommend starting at the $4 \mathrm{mg}$ dose and to potentially increase the dose with appropriate monitoring. While further high-quality trials that specifically address the vulnerable elderly population are needed, this study suggests that medically complex vulnerable elderly patients may safely benefit from pharmacotherapy and more specifically from fesoterodine.

\section{Beta-3 agonists}

Mirabegron, a beta-3 adrenoceptor agonist, is the first pharmacologic agent in its class to be approved for the treatment of OAB. It acts as a specific agonist of the beta-3 adrenoceptor on the human detrusor, leading to active relaxation. This occurs during the storage phase, and thus increases bladder capacity without affecting voiding ability. ${ }^{30}$

Wagg et $\mathrm{al}^{16}$ performed a prospective subanalysis of three 12-week, randomized Phase III trials in order to evaluate the efficacy and tolerability of mirabegron in subgroups of patients aged older than 65 years and older than 75 years. They found that in these two older age cohorts, mirabegron $25 \mathrm{mg}$ and $50 \mathrm{mg}$ once daily reduced the frequency of incontinence episodes and number of micturitions from baseline to last visit when compared with placebo. There appeared to be no loss of efficacy with age. In the group aged older than 65 years, hypertension, nasopharyngitis, and urinary tract infection were the most common treatment-emergent adverse events and occurred in $9.9 \%, 4.1 \%$, and $3.1 \%$ of patients, respectively. These were the most common adverse events in the group older than 75 years, in addition to headache, dry mouth, and extremity pain. However, over a one-year period, the incidence of the most common treatment-emergent adverse events was similar between both doses of mirabegron as placebo. Compared with tolterodine, an antimuscarinic agent, the incidence of dry mouth was six-fold less with mirabegron.

While the above subgroup analysis reports on two cohorts of older OAB patients, it does not mention their comorbidities or frailty status. The effect of renal or hepatic impairment on the pharmacokinetics of mirabegron was evaluated in a small open-label, single-dose, and parallel-group study. ${ }^{31}$ Mirabegron was generally well tolerated in subjects with mild, moderate, or severe renal or hepatic impairment. The pharmacokinetic changes observed in subjects with mild 
or moderate renal impairment and those with mild hepatic impairment were of small magnitude and likely clinically insignificant.

\section{Neuromodulation}

The fact that more than a century of developments in therapy aimed at detrusor suppression has not produced routinely well tolerated and efficacious therapies attests to the inadequacies of a paradigm equating $\mathrm{OAB}$ with overactivity of the detrusor. More recently, the contributions of sensory abnormalities have been considered, and efforts to directly modulate signaling between brain and bladder have been developed. Electrical neuromodulation via sacral or posterior tibial nerve stimulation has been shown to be an effective intervention for $\mathrm{OAB}$ in patients unresponsive to pharmacologic therapy. ${ }^{32}$ While some reviewers have questioned its efficacy in the frail elderly, ${ }^{33}$ recent evidence suggests that these technologies may be more effective than originally anticipated in this population. ${ }^{34}$ The ability to manipulate the programmer may be a consideration with sacral nerve stimulation; however, anecdotally, some practitioners have used these systems in the supervised frail elderly unable to operate the systems themselves (T Griebling, SUFU, verbal communication, 2014). Thorough documentation is necessary to ensure that the devices are not neglected should a trusted caregiver depart.

\section{Botulinum toxin}

Botulinum toxin is now approved by the US Food and Drug Administration for use in adults with idiopathic refractory $\mathrm{OAB}$, and the literature is replete with trials evaluating the efficacy and safety of botulinum toxin in the general population. As with the previously mentioned therapies, there is a paucity of evidence supporting the use of botulinum toxin $\mathrm{A}$ for $\mathrm{OAB}$ in the vulnerable elderly. Botulinum toxin exerts its effect upon the detrusor muscle by binding to membrane receptors on cholinergic neurons. The toxin is then internalized where it inhibits release of acetylcholine at the presynaptic cholinergic terminals. It is thought to inhibit striated and smooth muscle contraction and to suppress bladder afferent activity. ${ }^{35}$

One small study evaluated the efficacy of botulinum toxin $\mathrm{A}$ for refractory $\mathrm{OAB}$ in patients aged 75 years and older. ${ }^{36}$ Fourteen patients had idiopathic OAB and seven had neurogenic $\mathrm{OAB}$. Comorbidity, cognitive, and functional status were not reported. Following the first injection of $200 \mathrm{U}$, $76.2 \%$ of patients in the cohort reported $\geq 50 \%$ improvement in number of voids and/or pads used per day. Mean time to deterioration was 7.12 months.

Known complications associated with intradetrusor botulinum toxin include hematuria, urinary tract infection, and urinary retention. In the above-mentioned study, ${ }^{36}$ there were two reported urinary tract infections and no episodes of urinary retention. However, this was a small study that included only 21 patients. The potential risks of urinary retention may limit the feasibility of therapy in the vulnerable elderly patient who might not be able to perform or may not tolerate intermittent catheterization. The attenuation of response may also limit its use in the vulnerable elderly, who may not easily tolerate repeat injection and/or anesthesia.

\section{Conclusion}

$\mathrm{OAB}$ is problematic in the general population. As a symptom complex, it is often mistaken for a pathophysiologic process, an assumption that may sometimes be functionally valid in a medically uncomplicated population. However, the vulnerable elderly present a much more complex picture, with baseline frailty and multiple comorbid conditions. Further complicating the picture is the complex understanding of symptomatology, which must address patient goals and motivations as well as the expectations of caretakers. These multiple levels of perception, function, expectations, treatment efficacy, and risk must be tailored to the individual patient. While the vulnerable elderly patient may often have urinary tract dysfunction, $\mathrm{OAB}$ and urge urinary incontinence must be understood as a geriatric syndrome and in the context of medical and functional baseline and precipitating risk factors. Expectations and goals must be tailored to the resources of the vulnerable elderly patient or their caregiver, and care must be coordinated with the patient's other medical care providers. Management of OAB in the vulnerable elderly often poses significant management challenges. Nonetheless, with a thoughtful approach and an aim towards future research specifically for this population, significant reductions in morbidity and mortality as well as improved health-related quality of life are possible.

\section{Disclosure}

The authors report no conflicts of interest in this work.

\section{References}

1. Schneider T, Marschall-Kehrel D, Hanisch JU, Michel MC. Do gender, age or lifestyle factors affect responses to antimuscarinic treatment in overactive bladder patients? Int J Clin Pract. 2010;64(9):1287-1293.

2. Irwin DE, Milsom I, Hunskaar S, et al. Population-based survey of urinary incontinence, overactive bladder, and other lower urinary tract symptoms in five countries: results of the EPIC study. Eur Urol. 2006;50(6):1306-1314. 
3. Stewart WF, Van Rooyen JB, Cundiff GW, et al. Prevalence and burden of overactive bladder in the United States. World J Urol. 2003;20(6): 327-336.

4. Brown JS, McGhan WF, Chokroverty S. Comorbidities associated with overactive bladder. Am J Manag Care. 2000;6(11 Suppl):S574-S579.

5. Wagner TH, Hu TW, Bentkover J, et al. Health-related consequences of overactive bladder. Am J Manag Care. 2002;8(Supp1 19): S598-S607.

6. Brown JS, Vittinghoff E, Wyman JF, et al. Urinary incontinence: does it increase risk for falls and fractures? Study of Osteoporotic Fractures Research Group. J Am Geriatr Soc. 2000;48(7):721-725.

7. Morris V, Wagg A. Lower urinary tract symptoms, incontinence and falls in elderly people: time for an intervention study. Int J Clin Pract. 2007;61(2):320-323.

8. Coyne KS, Sexton CC, Irwin DE, Kopp ZS, Kelleher CJ, Milsom I. The impact of overactive bladder, incontinence and other lower urinary tract symptoms on quality of life, work productivity, sexuality and emotional well-being in men and women: results from the EPIC study. BJU Int. 2008;101(11):1388-1395.

9. Anger JT, Saigal CS, Madison R, Joyce G, Litwin MS. Increasing costs of urinary incontinence among female Medicare beneficiaries. $J$ Urol. 2006;176(1):247-251.

10. Litman HJ, McKinlay JB. The future magnitude of urological symptoms in the USA: projections using the Boston Area Community Health survey. BJU Int. 2007;100(4):820-825.

11. Abrams P, Cardozo L, Fall M, et al. The standardisation of terminology in lower urinary tract function: report from the standardisation sub-committee of the International Continence Society. Urology. 2003;61(1):37-49.

12. Gormley EA, Lightner DJ, Burgio KL, et al. Diagnosis and treatment of overactive bladder (non-neurogenic) in adults: American Urological Association/SUFU guideline. J Urol. 2012;188(Suppl 6):2455-2463.

13. Das R, Buckley J, Williams M. Dimensions of sensation assessed in urinary urgency: a systematic review. $J$ Urol. 2013;190(4):1165-1172.

14. Inouye SK, Studenski S, Tinetti ME, Kuchel GA. Geriatric syndromes: clinical, research, and policy implications of a core geriatric concept. J Am Geriatr Soc. 2007;55(5):780-791.

15. DuBeau CE, Kuchel GA, Johnson T 2nd, Palmer MH, Wagg A; Fourth International Consultation on Incontinence. Incontinence in the frail elderly: report from the 4th International Consultation on Incontinence. Neurourol Urodyn. 2010;29(1):165-178.

16. Wagg A, Cardozo L, Nitti VW, et al. The efficacy and tolerability of the beta3-adrenoceptor agonist mirabegron for the treatment of symptoms of overactive bladder in older patients. Age Ageing. March 14, 2014. [Epub ahead of print.]

17. Kuchel GA, Moscufo N, Guttmann CR, et al. Localization of brain white matter hyperintensities and urinary incontinence in community-dwelling older adults. J Gerontol A Biol Sci Med Sci. 2009;64(8):902-909.

18. Sakakibara R, Panicker J, Fowler CJ, et al. Is overactive bladder a brain disease? The pathophysiological role of cerebral white matter in the elderly. Int J Urol. 2014;21(1):33-38.

19. Smith PP, Wolfson LI, Kuchel GA. The neurourology of urinary symptoms in older adults. Virtual Editions, BJUI Online; 2011. Available from: http://www.bjui.org/ContentFullItem.aspx?id=665\&Lin kTypeID=1\&SectionType=1\&title=The-Neurourology-of-UrinarySymptoms-in-Older-Adults. Accessed July 4, 2014.
20. Becher K, Oelke M, Grass-Kapanke B, et al. [Improving the health care of geriatric patients: management of urinary incontinence: a position paper]. Z Gerontol Geriatr. 2013;46(5):456-464. German.

21. Lucas MG, Bosch RJ, Burkhard FC, et al. EAU guidelines on assessment and nonsurgical management of urinary incontinence. Eur Urol. 2012;62(6):1130-1142.

22. Gerretsen P, Pollock BG. Drugs with anticholinergic properties: a current perspective on use and safety. Expert Opin Drug Saf. 2011;10(5): 751-765.

23. Callegari E, Malhotra B, Bungay PJ, et al. A comprehensive non-clinical evaluation of the CNS penetration potential of antimuscarinic agents for the treatment of overactive bladder. Br J Clin Pharmacol. 2011;72(2):235-246.

24. Cetinel B, Onal B. Rationale for the use of anticholinergic agents in overactive bladder with regard to central nervous system and cardiovascular system side effects. Korean J Urol. 2013;54(12): 806-815.

25. Wagg A, Verdejo C, Molander U. Review of cognitive impairment with antimuscarinic agents in elderly patients with overactive bladder. Int J Clin Pract. 2010;64(9):1279-1286.

26. Yamada S, Maruyama S, Takagi Y, Uchida S, Oki T. In vivo demonstration of M3 muscarinic receptor subtype selectivity of darifenacin in mice. Life Sci. 2006;80(2):127-132.

27. Kay G, Crook T, Rekeda L, et al. Differential effects of the antimuscarinic agents darifenacin and oxybutynin ER on memory in older subjects. Eur Urol. 2006;50(2):317-326.

28. Zinner N. Darifenacin: a muscarinic M3-selective receptor antagonist for the treatment of overactive bladder. Expert Opin Pharmacother. 2007;8(4):511-523.

29. Dubeau CE, Kraus SR, Griebling TL, et al. Effect of fesoterodine in vulnerable elderly subjects with urgency incontinence: a double-blind, placebo controlled trial. J Urol. 2014;191(2):395-404.

30. Imran M, Najmi AK, Tabrez S. Mirabegron for overactive bladder: a novel, first-in-class beta3-agonist therapy. Urol J. 2013;10(3):935-940.

31. Dickinson J, Lewand M, Sawamoto T, et al. Effect of renal or hepatic impairment on the pharmacokinetics of mirabegron. Clin Drug Investig. 2013;33(1):11-23.

32. van Kerrebroeck PE, van Voskuilen AC, Heesakkers JP, et al. Results of sacral neuromodulation therapy for urinary voiding dysfunction: outcomes of a prospective, worldwide clinical study. J Urol. 2007;178(5):2029-2034.

33. McAchran SE, Daneshgari F. Sacral neuromodulation in the older woman. Clin Obstet Gynecol. 2007;50(3):735-744.

34. White WM, Mobley JD 3rd, Doggweiler R, Dobmeyer-Dittrich C, Klein FA. Sacral nerve stimulation for refractory overactive bladder in the elderly population. J Urol. 2009;182(4):1449-1452.

35. Apostolidis A, Dasgupta P, Denys P, et al. Recommendations on the use of botulinum toxin in the treatment of lower urinary tract disorders and pelvic floor dysfunctions: a European consensus report. Eur Urol. 2009;55(1):100-119.

36. White WM, Pickens RB, Doggweiler R, Klein FA. Short-term efficacy of botulinum toxin a for refractory overactive bladder in the elderly population. J Urol. 2008;180(6):2522-2526.
Research and Reports in Urology

\section{Publish your work in this journal}

Research and Reports in Urology is an international, peer-reviewed, open access journal publishing original research, reports, editorials, reviews and commentaries on all aspects of adult and pediatric urology in the clinic and laboratory including the following topics: Pathology, pathophysiology of urological disease; Investigation and treatment of

\section{Dovepress}

urological disease; Pharmacology of drugs used for the treatment of urological disease. The manuscript management system is completely online and includes a very quick and fair peer-review system, which is all easy to use. Visit http://www.dovepress.com/testimonials.php to read real quotes from published authors. 